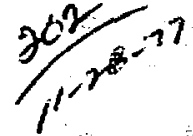 \\ LA-6920-N}

Mariual

UC-32

Issued: October 1977

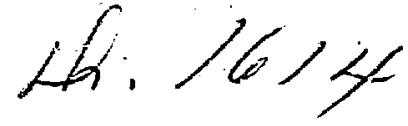

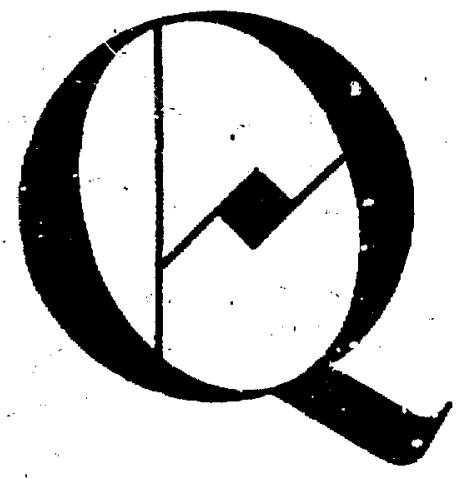

\title{
A System for
}

\section{Hišăogram Entry, Retrieval, and Plotting}

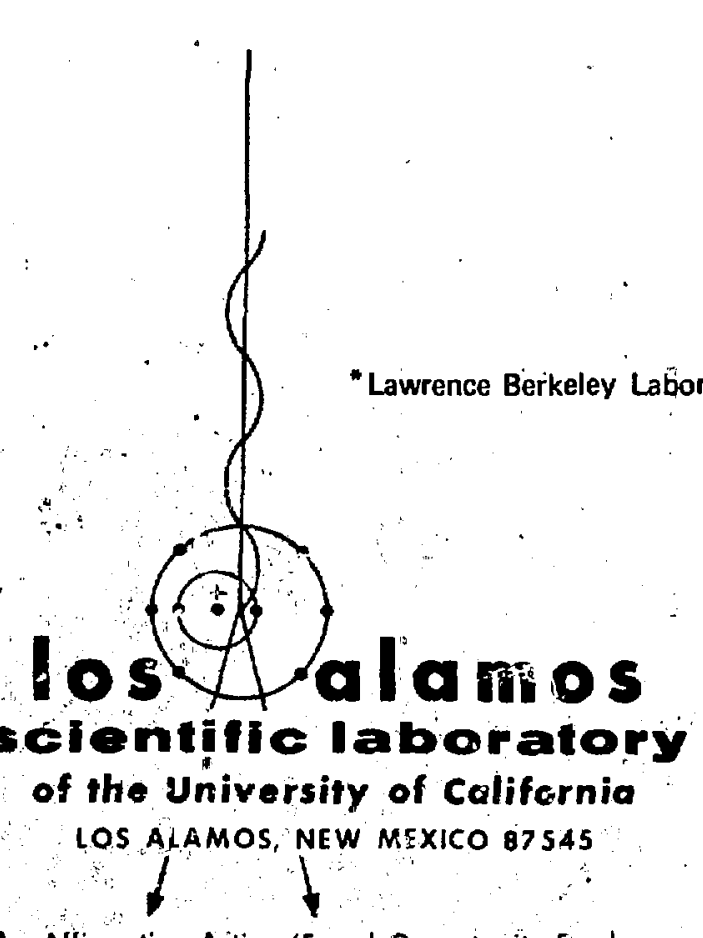

\author{
Martin Kellogg \\ Jeffrey M. Gallup* \\ Sally Shlaer* \\ Nancy Spencer
}

An Allirmative Action /Equal Opporiunity Employer 
This manual is one of a series describing the components of the Q general-purpose data-acquisstion system. This system, developed at the clinton $P$. Anderson Meson Physics racility, is designed to be useful in a variety of experimental situations for managing the acquisition and display of experinental data. It runs on the Digital Equipment corporation (DEC) PDP-11 family of computers running the RSX-1ID operating system and equipped with Microprogrammed Branch Drivers (MBDs) and CAMAC branch highway systems.

The $Q$ system is designed to allow high-speed data acquisition and recording. It is ariven primarily by the occurrence of events in the experimental apparatus. It is modular in construction, and is easy to use, understand, and modify. Its components are an IABD program for data taking, an RSX handler for data recording and distribution, a control and analysis task (the analyzer task), a histogram entry and cotrieval system, histogram plotting and dot plotting packages, and an operator control system. One special piece of hardware is required: a CAMAC module for responding to experimental events.

The histogram management and plotting systems (but not the dot plotting) have beeil implemented under R.SX-11M as well as RSX-1ID.

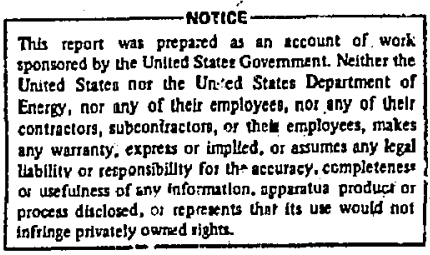


CONTENTS

1. INTRODUCTION . . . . . . . . . . ............. 1

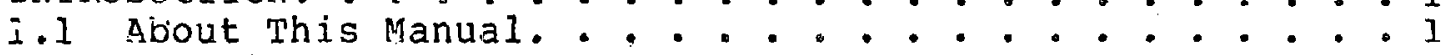

1.2 Overview . . . . . . . . . . . . . . . . . . . 2

1.3 Common Confusions. . . . . . . : . . . . . . . . . . 5

2.9 THE HISTOGRAM SYSTEM. - . . . - . . . . . . . . . . . . . . 5

2.1 IHEA: The Histogram Descriptor Array . . . . . . . . 5

2.2 Main Histogramming Subroutines . . . . . . . . . . . . 9

2.2.1 HISTSU: Histogram Creation (Setup) Subroutine. 9

2.2.2 HISTEN: Data Entry Subroutine .......... . 10

2.2.3 HISTRE: Histogram Retrieval Subroutine.... . .

2.3 Histcgram Utility subroutines. . . . . . . . . . . . . 12

2.3.1 HISTFL: Flush All Data Entries To Histogram . .12

2.3.2 HISTCL: Clear a Single Histogram... . . . . .13

2.3.3 HISTCA: Clear All Histograms. . . . . . . . . 3

2.3.4 HISTDE: Delete a Single Histogran . . . . . . .14

2.3.5 HISTDA: Delete All Histograms . . . . . . . 15

2.4 Wriring and Building a Histogramming Task. . . . . . .15

2.4.1 Writing the Task. ............... . . 15

2.4.2 Building the Task............... . . . . . . .

2.5 Keyboard Control of the Histogram system . ... . . . . 18

2.5.I HIS: List Histogram Descriptor Ariay

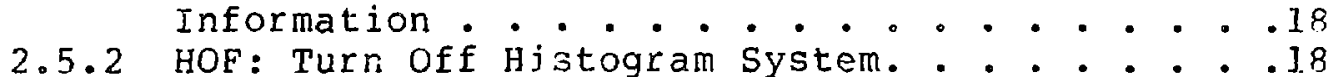

3.0 THE histogram plotting PACKage. . . . . . . . . . . . . . 19

3.1 HBE: Initialize the Disp?ay System . . . . . . . . . 19

3.2 HPL: Histogram Plot. . . . . . . . . . . . . . . 20

4.Ø THE DOT PLOTTING SYSTEM . . . . . . . . . . . . . . . . . . 22

4.1 Dot Plotting subroutines . . . . . . . . . . . . . . . 22

4.1.1 DOTMGE: Dot Plotting Management Subroutine. . .22

4.1.2 DOTOUT: Data Output Subroutine. . . . . . . .22

4.2 Writing and Building a Dot Plotting Task. . . . . . . 23

4.2.1 Writing the Task. . . . . . . . . . . . . 23

4.2.2 Building the Task............. . . 24

4.3 Keyboard Control of the System . . . . . . . . . . . 25

4.3.1 HDO: Dot Pיot Setup and Control . . . . . . . 25

ACKLOWLEDGMENTS . . . . . . . . . . . . . . . 28

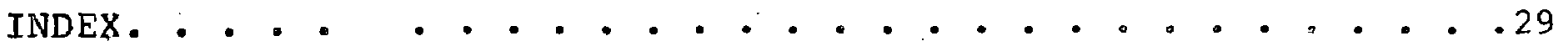


A SYSTEM FOR HISTOGRAM ENTRY, RETRIEVAL, AND PLOTTING

by

Martin Kellogg, Jeffrey M. Callup,

Sally Shlaer, and Nancy Spencer

\begin{abstract}
This manual describes the histogramming and dot plotting systems that rave been designed for use in connection with the $Q$ general-purpose data-acquisition system. These systems allow for the creation of histograms, the entry, retrieval, and plotting of histogrammed data, and the dynamic display of scatter plots as data are acguired. Although the systems are designed for use with $Q$, they can also be used as a part of other applications.
\end{abstract}

\title{
I. 0 INTRODUCTION
}

\subsection{About this Manual}

This manual describes the histogramming and dot plotting systems that have been developed for RSX-11. These systems have been developed and used primarily with the $Q$ data-acquisition system; however, any iask that runs under the RSX-11 system can make use of the Eacilities provided.

This manual describes what the user must do in order to utilize the 
system. It assumes that the reader is fairly fluent in FORTRAN and is familiar with the RSX-11 operating system; it alsu assumes a reasonible acquaintance with the concepts of histogramming.

\subsection{Overview}

The histogram entry and retrieval system and its associated plotting packages provide the capability to create, manipulate, and displav one- and two-dimensional histograms. Histograms are referred to by name and owner or by number, much in the manner uf files; they are maintained in central memory; and they can be displayed on a Tektronix 4000 series graphics terminal.

A subroutine creates a histogram and allocates memory* for it outside the calling task. At the same time, the user specifies the properties of each histogram and declares the form of the data to be histogrammed. Subroutine calls from the user task enter data into the histogram; during this process, the system automatically extracts the required date. and increments the appropriate histogran. bin. Other subroutine calls clear or delete histograms, or retrieve histogrammed data. Pertinent properties of the histogram system are as follows.

1. A maximum of 125 histograms may exist at cne time.

2. Histogramming takes place only in high-speed memory. At present, disk histograms are not supported.

3. A mi :imum of $64 \mathrm{~K}$ words can be allocated to histogram storage.

4. Overflows are handled aut smatically.

5. Any mix of one- and two-dimensional kistograms is allowed.

6. The system is independent of the remainder of the $Q$ data-acquisition system.

7. Nultilask access to histograms is supported.

8. Histograming is controlled entirely by user-written tasks.

Functionally, histogram system operation is shown in Fig. 1. The

*To circumvent the RSX-11 restriction of a $32 \mathrm{~K}$ riaximum task size and to enatle multitask access to the histograms, memory for histograms is allocated from the FiSX-11 GEN task partition. 
plotting package refers only to histograms built by the histogram system. It is a collection of tasks that run concurrently with the real-time data histogramming. The user defines the plotting style for each histogram by typing instructions to a control task. He can then request the plotting of a particular histogram with little additional keyboard work. Plotting parameters include log or lincar scale specification, bin range, character or vector plotting, and plot labeling.

The pr.perties of the plotting package are summarized as follows.

1. Any Tektronix 4000 series graphics terminal can be used as a plotting device.

2. Only histograms built by the histogram system can be displayed.

3. One-dimensional. stored histograms can be plotted in several formats by keyboard control.

4. At present, two-dimensional stored histograms are not supported by. the plotting package (but see dot plotting, Sec. 4.0).

5. The user need supply no code; however, the structure of the histogram system allows the easy replacement or extension of the plotting package if special user displays are required.

Functionally, the plotting package operation is shown in Fig. 2 .

The dot plotting system provides the capability to create a live, two-dimensional scatter plot on any Tektronix $400 \emptyset$ series graphics terminal. The terminal screen provides the memory for this special case of two-dimensional histogram plotting. A subroutine called by the user task initializes the dot plotting facility and enables keyborad communication with the user task for plot control. Subroutines called by the user task outpu'c points directly as data are acquired. The user interacts through the keyboard with a task that effects dot-plotting specification and control. Only one active scatter plot can be made at one time; however, the user can switch back and forth between several specified plots. Dot plotting. is done in real time with live data.

Functionaliy, the dot plotting system operation is shown in Fig. 3. 


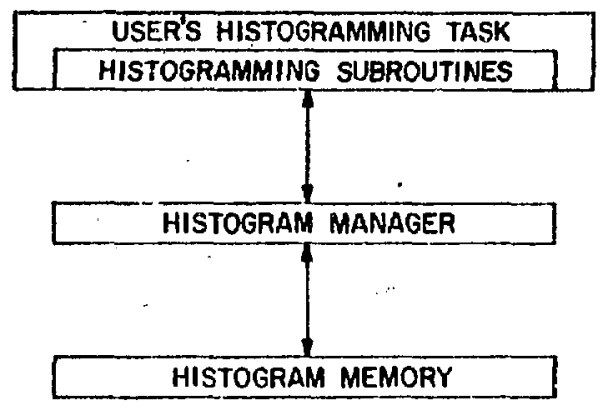

Fig. i.

FUNCTIONAL DIAGRAM OF THE HISTOGRAM SYSTEM.

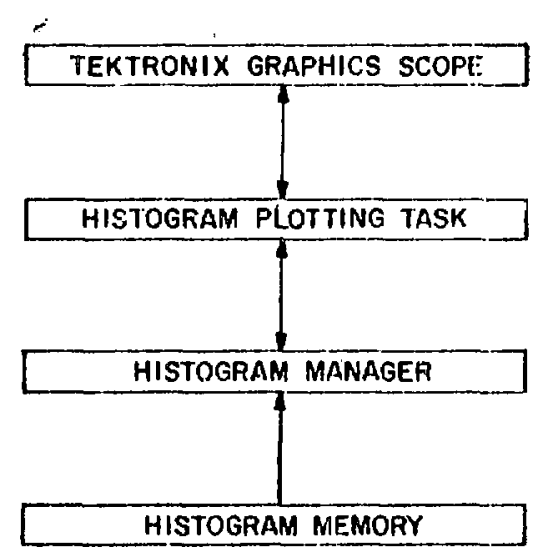

Fig. 2.

FUNCTIONAL DIAGRAM OF THE HISTOGRAM PLOTTING PACKAGE. 4

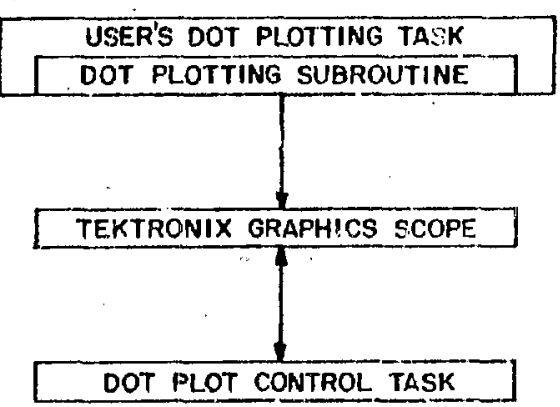

Fig. 3.

FUNCTIONAL DIAGRAM OF THE COOT PIOTTING SYSTEM. 


\subsection{Common Confusions}

Before proceeding, we would like to make clear the differences between a histogram, a histogram plot, and a dot plot.

A histogram is $\varepsilon$ imply ar array containing tallies representing how many times a certain data value has occurred. A histogram is not a picture, although a picture can be drawn to represent its contents.

A histogram plot is a snapshot that shows in graphic form the contents of a histogram. A histogram plot is not "? : ve" ; repeated snapshots may be taken to show the developments of the histogram over time, but each plot represents the state of the histogram as it appeared at a single instant in the recent past. Because the contents of the histogram and its plotted representation are distinct, the histogram data can be replotted with different parameters, such as vertical scales or plot characters.

There would probably be less confusion between histograms and histogram plots had dot plotting never been invented. A dot plot is a most peculiar animal indeed, being neither histogram nor histogram plot, but possessing properties of both. A dot plot is a live represencation of points (usually experimental data) in two-dimensional space. Hence, a dot plot is a tally system, where the "counts" are bright spots on the face of a storage CRT, which serves as a form of "histogram memory." This memory, and hence its contents, can be read out only by the human eye. Therefore, anything that has been "histogrammea" in this way is not available for further processing. In particular, a dot plot cannot be retrieved after erasing and cannot be replotted with new parameters.

\section{2. $\emptyset$ THE HISTOGRAM SYSTEM}

\subsection{IHDA: The Histogram Descriptor Array}

A 16-bit integer histogram descriptor array (IHDA) has a central role in histogram reference. The user describes or identifies a histogram to the system and receives status information from the system through IHDA. The system uses IHDA only during subroutine execution; at other times, the user is free to change it. The user need not maintain a descriptor array for each histogran!, because the information in it is maintained internally by the system once the histogram has been created.

The first five words in IHDA identify the histogram. 
IHDA(1) - IHDA(3): histogram name

The name consists of six ASCII characters from the RAD50 subset (A-Z, $\emptyset-9$, period, space, dollar sign); embedded spaces are not allowed but trailing spaces are allowed.* If $\operatorname{IHDA}(1)=\emptyset$, the histogram is identified by its number [see IHDA (5) ] .

The following histogram names are legal:

HISTI0

TDC

DELE.I

The following names are illegal:
H IST
Has an embeddeõ blank.
$\mathrm{DE} / \mathrm{DX}$
The / is not in the RAD50 subset.
C. 1.2
This name is legal; however, the display system cannot access it because it has two periods.
$A \$ \times 9$
This name is legal; however, the display system cannot access it because it has a dollar sign.

IHDA (4): histogram owner

The owner specifies is user identification code (UIC) consisting of octal group and user numbers [UICG,UICU] so that

$$
\operatorname{IHDA}(4)=U I G * 256+U I C U
$$

For example, if the owner's UIC is $[261,3]$, we have, in FORTRAN syntax,

$$
\operatorname{IHDA}(4)=" 261: 256+" 3
$$

and the octal value of IHDA(4) is 130403 .

IHDA (5): histogram number

This number is assigned by HISTSU when the histogram is created. Numbers are assigned sequentially starting with one. When a histcgram is deleted, its number becomes available for reassignment. Identification by number provides faster access to the histogram than identification by name and owner; it is allowed at any time after the

* If a histogram is to ke accessed by the display system, its name may not contain more than one period, and no more than three nonblank characters may follow the period. Furthermore, the name may not contain a dollar sign. 
histogram has been created. Identification by number is not allowed by HISTSU, becalise HISTSU assigns the number when it creates the histogram. HISTEN requires identification by number. The maximum number of histograms permitted at one time is 125 .

The following words are used only during histogram creation and retrieval:

IHDA (6): number of parameters

Tris value must be one or two and specifies the dimension of the histogram.

IHDA (7) - IHDA (11) describe the first (or only) histogram parameter. They specify to HISTEN how to process the data block and determine which bin to increment.

IHDA(7): data index (I1)

IHDA (8): mask (MI)

Il and $M I$ select the item in the data block to be histogrammed. Il is the offset in words from the beginning of the block to the word containing the item, so that Il=0 refers to the first word in the block. A logical. AND. between $M I$ and the word selected by Il extracts a field containing the number to be histogrammed. This number is unsigned, and its low-order bit corresponds to the rightmost "one" bit in $M I$; it is referred to below as XI. For example, suppose that IHDA(7.) $=2$, IHDA $(8)=" 370$, and the event data block in octal form is

179723

Then the third data item, 170723, is selected and masked to yield 320 in octal. After the shift implied by IHDA(8), we have $\mathrm{Xl}=32$ in octal.

A rnask of $\emptyset$ would not result in a meaningful histogram and hence is invalid. A nask of -1 (177777 octal.) will cause the specified data item to be histogrammed exactiy as it appears in the data block. In the above example, the third data item (170723) would be selected and histogrammed without change.

Data values to be histogrammed are treated as unsigned 16-bit numbers ranging from to 177777 (octal) $=2 * * 16-1$. This differs from the signed twos-complement representation of integers used by FORTRAN, where numbers range from 100000 
(octal) $=-2 * * 15$ through $177777=-1$ and $\emptyset$ to $77777=$ $+2 * * 15-1$. For this reason, negative data values cannot be histogrammed directly. First, they must be biased by adding a positive number to each value so that only positive values are histogrammed. For example, suppose we wish to histogram data values that 1 ie in the range -256 to +255 . By adding 256 to each value, we convert the range to $\emptyset$ to 511. The values may then be histogrammed into 512 bins by using a lower limit of $\emptyset$ and a bin size of 1 .

IHDA(9): lower limit (LI)

The lower limit is treated as an unsigned 16-bit number (see IHDA(7) and IHDA(8) above). This is the lowest data value that can be tallied into this histogram without underflow.

IHDA (10): bin size (SI)

IHDA (11): number of bins (N1)

The following formula is applied to the data value $x 1$ to determine which bin $B$ to increment:

$B=\operatorname{MIN} \emptyset(\mathrm{N} 1, \operatorname{MAX} \theta(1,(\mathrm{X} 1-\mathrm{L} 1) / \mathrm{S} 1+1)$

Bins are numbered 1 through N1. Note that "underflowed" and "overflowed" bin numbers increment the lowest and highest bins, respectively.

IHDA (12)-IHDA (16) describe the histogram's second parameter; they must be present only if a two-dimensional histogram has been specified in IHDA $(6)$.

IHDA (12): data index (I2)

IHDA (13): mask (M2)

IHDA (14): lower limit (L2)

IHDA (15): biń size (S2)

IHDA (16): number of bins (N2)

For a two-parameter histogram, the following formulas

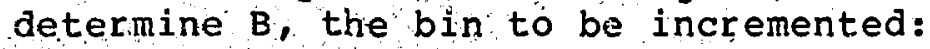

$B 1=\operatorname{MIN} \emptyset(N 1, \operatorname{MAX} \emptyset(1,(X 1-L 1) / S 1+1)$

$B 2=\operatorname{MINO}\left(\mathrm{N} 2, \operatorname{MAXO}\left(1,\left(X_{2}-L_{2}\right) / \mathrm{S} 2+1\right)\right.$ )

$B=((B 2-1) * N 1)+B 1$ 


\subsection{Main Histogramming Subroutines}

\subsubsection{HISTSU: Histcgram Creation (Setup) Subroutine}

Form:

CALL HISTSU ( IHDA, IERR )

In IHDA (Sec. 2.1) the caller provides the name and owner of the histogram, and specifies its dimensionality and the base and extent of each index. The histogram number is returned in IHDA(5) by HISTSU; it must be remembered for access through HISTEN. If IERR is returned as nonzero, cne of the following errors has occurred:

$$
\begin{aligned}
& \text { IERR }=1: \\
& \text { IERR }=2: \\
& \text { IERR }=3: \\
& \text { IERR }=4: \\
& \text { IERR }=5: \\
& \text { IERR }=6: \\
& \text { IERR }=7: \\
& \text { IERR }=8:
\end{aligned}
$$

Insufficient histogram memory is available. There is insufficient space in the histogram system dynamic memory.

No slot is available in the number table; that is, 125 histograms already exist. The number of parameters is not $l$ or 2 . Parameter information is invalid or internally inconsistent. The histogram name is not given or not valid. The histogram already exists. HISTSU cannot request the histogram management task. (Most likely the system is improperly configured.)

HISTSU does not clear the histogram that it creates. HISTCL (Sec. 2.3.2) or HISTCA (Sec. 2.3.3) must be called to clear the histogram before values are entered.

Example:

C THIS ROUTINE CREATES A ONE-DIMENSIONAL HISTOGRAM FOR SCALER

C DATA WITH THE NAME 'SCALER', OWNER $[122,24]$, WITH 1024 BINS

C AND A BIN SIZE OF 2. DATA VALUES WILL RANGE FROM $\square$ TO

C 2047 .

C

COMMON/DWD7/IHDRWD, IGATE, LATCH , ISCALR

DIMENSION IHDA (16)

C CREATE IHDA - HISTOGRAM DESCRIPTOR ARRAY

$\operatorname{IHDA}(1)=$ ' SC'

IHDA $(2)=$ ' $A L$ '

IHISTOGRAM NAME

$\operatorname{IHDA}(3)={ }^{\prime} \mathrm{ER}^{\prime}$

$\operatorname{IHDA}(4)=" 122 * 256+" 24$

! OWNER

$\operatorname{IHDA}(6)=1$

$\operatorname{IHDA}(7)=3$

!NUMBER OF PARAMETERS

$\operatorname{IHDA}(8)=" 177777$

! DATA INDEX

I MASK 

$\operatorname{IHDA}(9 ;=$
IHDA $(1 \emptyset)=2$
$\operatorname{IHDA}(11)=1024$
! LOWER LIMIT
!BIN SIZE
! NUMBER OF BINS
C SET UP HISTOGRAM
CALL HISTSU (IHDA, IERR)
IF (IERR.NE. D) GO TO 100
C SAVE HISTOGRAM NUMBER RETURNED BY HISTSU FOR USE BY HISTEN NUMH ST $=$ IHDA (5)
C. CLEAR THE HISTOGRAM
CALL HISTCL (IHDA)

2.2 .2 HISTEN: Data Entry Subroutine"

Form:

CALL HISTEN ( $N$, DATA)

HISTEN is called each time we wish to increment a histogram bin. $N$ is the histogram number (remembered from HISTSU time). DATA is the data block from whirh HISTEN extracts a data value; it calculates the number of the bin to be incremented from this value. See Sec. 2.1 for the algorithm used to determine which bin to increment. If $\mathrm{N}$ is out of range or specifies a nonexistent histogram, HISTEN does nothing.

Example :

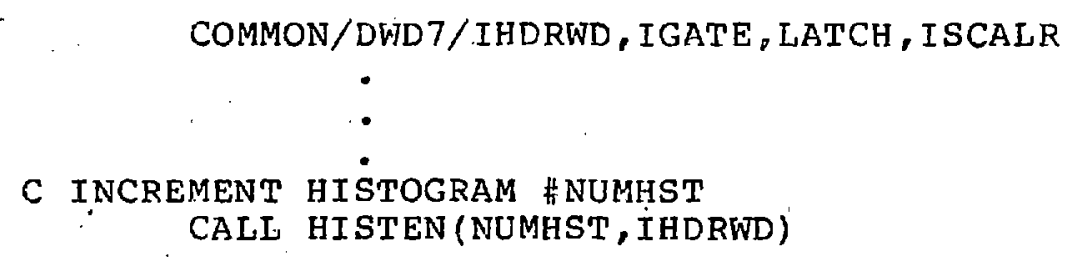

The example in sec. 2.2.1 shows how the histogram in this example was set up. Because speed of entry is important, and finding a histogram identified by its name and owner is a time-consuming process, HISTEN allows only identification by number.

\subsubsection{HISTRE: idistogram Retrieval Subroutine}

HISTRE retrieves the histogram descriptor array and, optionally, retrieves a specified subset of the histogram data. The histogram is specified by its name and owner or by its number. 
Form :

CALL HISTRE (IHDA, IERR, REJ,DATA, IL, IU, JL, JU)

IHDA (Integer Array): Histogram Descriptor Array. (Sec. 2.1). Descriptor array information is returned in IHDA if histogram name and owner or histogram number is specified in IHDA.

IERR (Integer): Error siatus:

IERR= 0 : No error.

IERR=1: The histogram does not exist or nu histogram specified.

IER.R=2: The bin limits (IL, IU, JL, JU) are incorrectly specified.

REJ (Rea1): Rejected event ccunt for the histogram. Events that cannot be histogrammed increment this count. priorities are such that for core histograms no events should be rejected.

DATA (Real array): Array into which the histogram data are retrieved.

IL (Integer): Lowest numbered bin to be retrieved for parameter 1 . IL must be at least $l$.

IU (Integer): Highest numbered bin to be retrieved for parameter 1. IU must be equal to or greater than IL.

JL (Integer): Lowest numbered bin to be retrieved for pacameter 2. JL must be at least 1 .

JU (Integer): Highest numbered bin to be retrieved for parameter 2. JU must be equal to or greater than JL.

The following points should be kept in mind.

1. IHDA and IERR must be specified and, if the DATA argument is present, then REJ, IL, and IU must also be specified.

2. For a two-parameter histogram, JL and JU default to 1 if they are not specified.

3. The order of retrieval in the two-parameter case (using "implied DO" notation) is ( $(B I N(I, J)$, $I=I L, I U), J=J L, J U)$.

4. It is permissible to attempt to retrieve bins that do not exist in the histogram. Such bins are assumed to contain zeros. 
Examples:

To cetrieve IHDA for a particular histogram number:

$$
\begin{aligned}
& \operatorname{IHDA}(1)=0 \\
& \operatorname{IHDA}(5)=\text { NUMHST }
\end{aligned}
$$

C RETRIEVE HISTOGRAM DESCRIPTOR FOR HISTOGRAM

C NUMBER WUMHST

CALL HISTRE (IHDA, IERR)

To retrieve IHDA and a subset of the histogram data for a particular one-parameter histogram:

$$
\begin{aligned}
& \operatorname{IHDA}(1)=\text { 'SC' } \\
& \operatorname{IHDA}(2)=\text { 'AL' } \\
& \operatorname{IHDA}(3)=\text { 'ER' } \\
& \operatorname{IHDA}(4)=" 122 * 256+" 24
\end{aligned}
$$

C FLUSH HIS'IOGRAM EN'IRIES

CALL HISTFL

C RETRIEVE HISTOGRAM DATA

CALL HISTRE (IHDA, IERR, REJ,DATA, IL,IU)

-

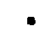

2.3 Histogram Utility Subroutines

2.3.1 HISTFL: Flush All Data Entries to Histograms

Form:

$$
r^{\circ} \because \text { HISTFL }
$$

HISTFL es that all buffers internal to the histogramming system emptied so that all histograms are updated properly. A task that uses the histogramming system should call HISTFL before it retrieves a histogram (HISTRE) or before it exits. 


\subsubsection{HISTCL: Clear a Single Histogram}

Form:

CALL HISTCL ( IHDA)

HISTCL sets the contents of a singie hiscogram to zeros. Overflow entiies associated with the histogram are removed from the overflow table. The histogram to be cleared is specified by IHDA (Sec. 2.1), and it can be identified either by its name and owner ir by its number. If the specified histogram does not exist, HISTr: does nothing.

HISTCL should always be called to clear a newly created histogram, since the contents of such a histogram are not defined.

Examples:

To clear a histogram by its name and owner:

$$
\begin{aligned}
& \operatorname{IHDA}(1)=' S C ' \\
& \operatorname{IHDA}(2 .)=\text { 'AL' } \\
& \operatorname{IHDA}(3)=\text { 'ER' } \\
& \operatorname{IHDA}(4)=" 122 * 256+" 24
\end{aligned}
$$

C CLEAR HISTOGRAM [122,24] SCALER

CALI, HISTCL (IHDA)

To clear a histogram by its number:

$$
\begin{aligned}
& \operatorname{IHDA}(1)=0 \\
& \operatorname{IHDA}(5)=\text { NUMHS'!! }
\end{aligned}
$$

C CLEAR HISTOGRAM NUMBER NUMHST

CALL HISTCL ( IHDA)

\subsubsection{HISTCA: Clear All Histograms}

Form:

CALL HISTCA ( IOWNER )

HISTCA clears all histograms belonging to IOWNER. IOWNER must match the owner number that was specified in IHDA(4) (Sec. 2.1) when the histograms were created. If no histogram belongs to the specified owner, HISTCA does nothing. 
Example:

CALL HISTCA $(" 122 * 256+" 24)$

2.3.4 HISTDE: Delete a Single Histogram

Form:

CALL HISTDE ! IHOA)

HTSTDE deletes a single histogram, freeing the resources allocated to it. The histogram an be deleted is specified by IHDA. The histogram may be identifie-. hy its name and owner or by its number. If the specified hictogram does not exist, HISTDE does nothing.

Examples:

To delete a histogram by its name and owner:

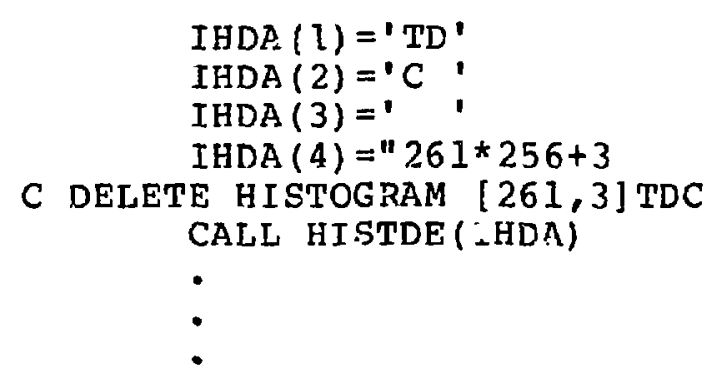

To delete a hiutogram by its number:

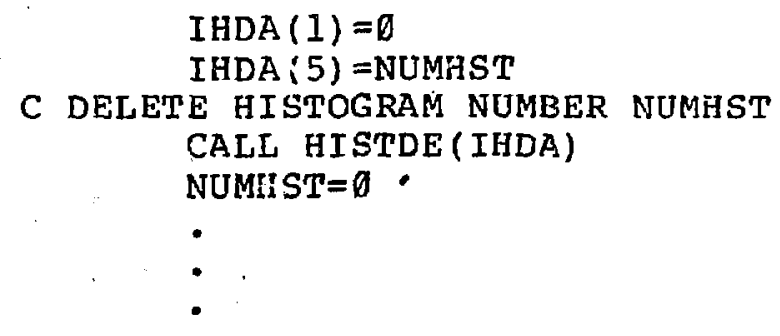

Note: Because the number that belonged to the deleted histogram will be reassigned when a nev histogram is created, the user should arrange to "undefine" the histogram number associated with the histogram that was just deleted. This is the purpose of the line NUMHST $=0$ in. the example above. 
2.3.5 HISTDA: Delete All Histograms

Form:

CALL HISTDA ( IOWNER )

HISTDA deletes all histograms belorging to IOWNER. IOWNER must match the owner number that was specified in IHDA(4) when the histograms were created. If no histogram belongs to the specified owner, HISTDA does nothing.

Example:

\author{
IOWNER $=" 261 * 256+3$ \\ C DELETE ALL HISTOGRAMS BELONGING TO [261,3] \\ CALI. HISTDA (IOWNER)
}

2.4 Writing and Building a Histogramming Task

\title{
2.4.1 Writing the Task
}

Although the histogram system was developed in conjunction with the Q data-acquisition system, not only a $Q$ analyzer, but any task, can use the facilities provided. "Because a histogram is identified by buth name and UIC, a true multiuser capability is provided.

The following puints should be kept in mind.

1. Histograin setup (HISTSU) should be performed in a section of the task or in a separate task that is executed only once. In a $Q$ analyzer, setup normally occurs in the background. Because histogram entries (UISTEN) must be specified by number, the number returned at setup time must 'be remembered for use later in incrementing histograms with HISTEN.

2. A maximum of 125 histograms can exist simultaneously; a user who requires more histograms can change this limit. It is set because the information that must be kept in. a system common area (HCOMl) for all histograms fits into 4096 words of memory if a maximum of 125 histograms is allowed. All tasks that access histograms must map to this system common, so we have decided to keep the memory to a size that can be inapped, with a single mapping: register. 
3. Memory is allocated to histograms outside the task that requests setup. Therefore, any number of tasks can have access to the bistograms. This capability is used in the is istogram display system, where separate plotting tasks ran retrieve and plot histogram data. Once histogram alemory has been allocated by a call to IIISTS, it is not releasec until all histogr amming is terminated by foF. Histugram memory cannot be checkpointed and, once allocated, it remains fixed in core. Therefore, the possibility of core fragmentation exists. It is probably not a pro' em in a $Q$ environment, owing to a careful core-loadisg algorithm; however, it may be a problem when histograming is performed in a true multiuser situation.

4. Memory is allocated in $1 . \mathrm{K}$ blocks. For example, if one 100 -word histogram is set up, $1 \mathrm{k}$ of memory is allocated that can be used only for histogram storage. If another 256-word histogram were set up later, its memory would be allocated from the remainder of the $l \mathrm{k}$ block already claimed $b_{y}$ the histogram system.

Under RSX-11D memory is alloceted from the bottom of the GEN partition up. Under RSX-11M it is allocated from the top of the GEN partition down.

5. Eistograms are not cleared when thev are set up. They contain anything that lies ir the memory locations allocated to them. The user must clear the histograms, either when he sets them up or at some other appropriate time. For instance, in a $Q$ environment, the user mar wish to clear all histograms at the start of a run from task QSRUN.

6. In a $Q$ environment, histogram entries (HISTEN) normally are made from the analyzer's event processors. Entries must be made by the histogram number, which must be remembered from the time if setup (HISTSU).

7. If a task calls HISTEN, the task should call HISTFL before it exits. If a task is going to call HISTRE, the task should call HISTFL before it calls HISTRE... These precautions are necessary to insure that all histograms are updated. However, too-frequent calls to HISTEL reduce the rate at which entries can be made.

8. Histograms can be deleted by either HISTDE or HISTDA. When a histogram is deleted, its number is released and the information about the histogram kept ir the system common ficomi is cleared. However, the core memory allocated for the histogram is not returned to the RSX system. Instead, it is kept by the histogram system and will be reallocated to future histograms as they are set up. Fragmentation can occur in the histogram memory; its 
occurrence depends on the sizes of histograms set up, to replace those which have been deleted. The only way to return core memory to the RSX system is to turn off histouraming by running HOF (Sec. 2.5.2).

9. As long as histograms exist, the histogram manager task HSTMGE remains in memory. The user must not abort this task because it keeps track of the core memory allocated for histograms. If HSTMGE is aborted, the memory allocated for histograms will never be returned to the RSX system unless the system is rebootstrapped. Running HOl? causes HSTMGE to return the histogram memory to the RSX system and then to exit.

10. If the partition mapper task PMP distributed with $\Omega$ is run, the memory allocated for histograms will be labelled "lost".

\subsubsection{Building the Task}

Subroutines HISTSU, HISTEN, HISTRE, HISTFL, HISTCL, HISTCA, HISTDE。 and HISTDA are available in the library MDLIB, which is distributed with $Q$. MDLIB must be referenced in a user task-build file if the task calls any of these subroutines.

All histogramming subroutines use a data base contained in the system common or system global area.(SGA) HCOMl; therefore, all task builu files for histogram tasks must contain one of the following COMMON specifications:

or

$$
\text { COMMON=HCOMI : RW: } 5 \quad \text { (RSX-11D) }
$$

$$
\text { COMMON=HCOMI:RW: } 6 \quad \text { (RSX-1IM) }
$$

Example:

; [122, 24] HSTTST.BLD $\emptyset 6-M A Y-77$ BLD FILE FOR HISTOGRAM TEST

OU: $[122,24]$ HSTTST US: $[122,24]$ HSTTST $=$ IN $:[122,24]$ HSTTST

; REFERENCE MDLIB \& $)$ GET HISTOGRAMMING SUBROUTINES

$S Y:[1,1] \mathrm{MDL} I \mathrm{~B} / \mathrm{LB}$

LIBR=OTSCOR: RO

iLINK TO THE RESIDENT FORTRAN LIBRARY (RSX-1ID)

COMMON=HCOMI:RW: 5 ; LINK TC SYSTEM COMMON (RSX-1LD)

if

The pseudo-devices IN:, ou:, and LS: must be redirected to an appropriate disk. DKn: before this task build file is run. 


\subsection{Keyboard Control of the Histogram System}

\subsubsection{HIS: List Histogram Descriptor Array Information}

This command lists the histogram descriptor array information by number for all defined histograms 1-i25. Ijisted are histogram name, UIC information, parameter \#1 information [IHDA(6)-IHDA(11)], and, when appropriate, parameter \#2 information [IHDA (12) - IHDA (16)] .

Form:

MCR>HIS

\section{5 .2 HOF: Turn Off Histogrim System}

This command turns off the entire package and releases all memory reserved for histograms to the system.

Form:

MCR $>$ HOF

Because of the system structure, HOF must be run before any user-supplied histogram task can be removed from the system. An orderly shutdown procedure is as follows:

1. Exit or abort any active user task involved with histogramming.

2. $M C R>H O F$

3. MCR $>$ REM user task

HOF can be run safely when the histogram system is totally inactive. Because the histogram system may not have been shut down properly by a previous user, it is recommended that the user run HOF before he begins any histogramming, simply as a satiety precaution. However, because HOF terminates all histogramming, one should run it only after determining that no other user wishes to continue histograming. 


\title{
3.Ø THE HISTOGRAIS PLOTTINE PACKAGE
}

Two tasks are associated with histogram plotting.* HBE initializes the plotting system. HPL plots histog:ams and performs such functions as clearing histograms and changing a terminal's default histogram owner UIC.

\subsection{HBE: Initialize the Display System}

HBE initializes the plotting system for histograms and dot plots by removing all plot entries and setting the current terminal's default histogram UIC to the logged-in UIC. This task should be run once before either HPL or HDO.

Form:

MCR $>$ HBE

\begin{abstract}
*Historical note: Because this plotting facility was developed in conjunction with $Q$, only code likely to be useful in a variety of experimental situations has been developed. Effective plots and sketches can be expected to vary with the experiment, ranging from curve-fitting displays to particle trajectories traced against a background of the detector system. 'Although we cannot fulfill all needs in a general plotting facility, we have tried to provide support for the one case. for which there is large demand: oneparameter histogram display. Because there are many ways to present two-parameter histogram displays and no consensus on the best way, we do not provide such a facility at this time. There are plotting subroutines in MDLIB, which the user can combine with algc:ithms for such things as contour or isometric plots. Source code is available for the plot task HPL; this may prove helpful both as a model and in providing subroutine building blucks for user-designed plotting code. Cursor interaction, because of its experiment-dependent nature, is another feature that has not been supplied in the general package. For readers interested in adding this capability the source files of HFL may prove helpful when used with the CURSIS subroutine from MDLIB." See "MDLIB: System Subroutine Library," Los Alamos Scientific Laboratory Group MP-1 internal document(1977).
\end{abstract}


3.2 HPL: His'ogram Plot

HPL is the general-purpose histogram plotting task that allows the user to request display of any one-dimensional histsgram. Requests are entered through any keyboard; displays are made on any Tektronix 4000 series terminal on the syster.

Forms:

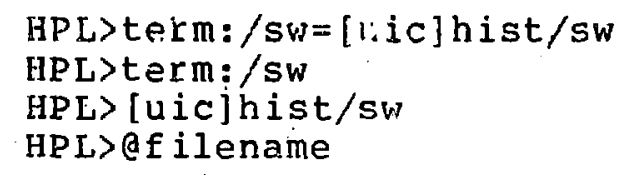

where term specifies a graphics terminal, [uic] a histogram owner, hist a histogram name, /sw a switch or switches, and filename the name of in indirect command file. Switches are optional; they either modify the plot or select auxiliary functions. If a line contains an equals sign, any acceptable switch can be used on either side of the sign.

The following defaults are used for omitted parts of the line:

term the current terminal

[uic] the default histogram UIC

hist the histogram last plotted

HPI plots histogram hist owned by [uic] on terminal term. The plotting defaults are

Linear $Y$ scale.

Full-scale $Y$ values selected automatically to plot all points on scale.

All bins in the histogram plotted.

Point plot using plotting dot.

A heading consisting of histogram name, date, and time.

These defaults can be overridden for the current plot (by switches) or changed for all subsequent plots of a given histogram.

The following switches are available. The first set selects auxiliary functions. Only one of the first set can be used at one time; its line cannot contain an equals sign.

1. /CL clear the specified histogram. If a UIC and no histogram name is given; clear all histograms owned by that UIC. (Confirmation is requested.) This switch affects the histogram itself; it is provided for convenience although it is not a plotting control operation.

2. /UI If a UIC is given, set the current terminal's default histogram UIC to it. If no UIC is given, print the 
default histogram UIC of the given terminal.

3. /-UI Remove the current terminal's default UIC setting.

4. /HI List all histograms set up for plotting at the given terminal. (NOTE: NOT YET IMPLEMENTED)

The second set of switches modifies the plot specifiad by the command line.

1. IE Erase the screen before plotting. The default is not to erase the screen $(/-E)$.

2. /o Overlay this plot on the previous plot, using the previous scaling.

3. /L Use a linear Y scale. (Default.)

4. /G Use a logarithmic Y scale.

5. /Y:high set the full-scale $Y$ value to 'high' and the bottom $Y$ value to zero (linear) or one (log). A decimal point and/or exponent can be given.

6. /Y:low:high set the full-scale $Y$ value to 'high' and the bottom $Y$ value to 'low.' 'Low' may be negative.

7. /-Y Use automatic Y scaling: (Default.)

8. /B:high set the highest bin to be displayed to 'high' and the lowest bin to one.

9. B:low:high set the lowest bin to be displayed to 'low' and the highest bin to 'high.'

10. /-B Display all bins in the histogram. (Default.)

11. /PP Do a point plot, plotting with a dot. (Default.)

12. /PP:char Do a point plot, plotting with the character 'char.' (Note: some characters, such as '=' or ', ', will cause syntax errors.)

13. Ní Lo a vector plot; that is, connect all plotted points with lines.

14. /BP Do a bar plot; that is, plot a vertical line from the $x$-axis to each point.

15. /HD:text: Use the text between the ns as a plot heading. Up to 27 characters of will be used. Colons may not appear in the text string. (NOTE: NOT YET IMP LEMENTED) 
16. /C Change the defaults for plottjing the given histogram. This.. switch is used in conjunction with other plot format switches. No plot is generated.

17. /CP Change the defaults for plotting the given histogram and generate a plot with the new defaults. This switch is used in conjunction with other plot format switches.

18. /A:n Add bins in groups of $n$ when plotting.

\subsection{THE DOT PLOTTING SYSTEM}

The dot plot system provides the capability of producing live two-dimensional scatter plots on any Tektronix 4000 series graphics terminal. The system consists of two subroutines that are called by the task wishing to create a det plot, plus an MCR task that allows keyboard control of the plotting. Only one dot plot can be active at one time; however, the user can stop a plot, then either restart it or start a new one.

4.1. Dot plotting subroutines

4.1.1 DOTMGE: Dot Plotting Management subroutine

Form:

CALL DOTMGE

A call to DOTMGE enables communication between the calling task and the MCR task HDo, so that plotting can be initialized and controlled through the keyboard. DOTMGE should be called once by the task wishing to create a dot plot. In a $Q$ analyzer, this call normally. is' made from the analyzer's background.

\subsubsection{DOTOUT: Data Output Subroutine}

Eorm:

CALL DOTOUT (IEVN, IEVBUF)

A call to DOTOUT is made when we want to output on the graphics Eerminal a dot based on the values of two numbers in the data buffer IEVBUF. "Whether a point is actually" output depends on 
whether dot plotting is currently enabled for "event" IEVN (a number between and 255). In a $Q$ analyzer, IEVN is normally the number $(6-31)$ of the event being processed and IEVBUF is the first word of the single event common block for that event. The number of the event for which plotting is to be enabled, offsets to the selected items in the data buffer (IEVBUF) to be plotted, and the axis limits are all specified by the HDO command (See Sec. 4.3.1).

4.2 Writing and Building a Dot Plotting Task

\subsubsection{Writing the Task}

The dot plotting facility can be used from any task that wants keyboard control of scatter plotting. The following points should be kept in mind when a dot plotting task is written:

1. DOTMGE must be called once from the task that will perform the dot plotting. DOTMGE specifies a receive AST to allow the task to receive control messages from HDO. (See the RSX-11 Executive Reference Manual for information about receive ASTS.) Therefore, any task that already specifies a receive AST will be incompatible with the dot plotting facility.* In a $Q$ environment, DOTMGE is called from the analyzer background.

2. DOTOUT is called from any location in the task where the user wants to plot a point on a scatter plot. Whether a dot will appear on the graphics scope is determined by whether a dot plot is active for the event number specified as a parameter to DoTOuT. In a $Q$ environment, DOTOUT is called from an event processor in the analyzer and the actual event number is specified as a parameter.

3. The dot plot control task HDo shares storage in the systeni global area HCOMI with the histogram display tasks. Information pertinent to a particular dot plot, such as

*If this restriction imposes unacceptable limitations, the user can obtain source code for DOTMGE and modify it to support a fan-out of control based on the sender task name or on the data sent. The source for the task QCNTRL provides a model for this type of structure, including cases where certain receives are serviced in preference to others. Any task that engages in tis isort of merriment should have a pretty firm understanding with all of its senders. 
the upper and lower plot bounds and the dot plotting task name, is kept in this area. Information pertinent to histogram plot defaults is kept in the same area. Currently, there is space for 35 entries, which can be all histogram display entries, all dot pict entries, or a combination of both. When a user tries to pecify a new set of histogram plot defaults or a new dot plot when 35 entries already exist, the space for the least recently used entry, either histogram plot or dot plot, is reused. By this action HDO may lose the information about a partichuar dot plot if that dot plot's entry space is reused. If this occurs the message "HDO--TASKNAME MUST BE SPECIFIED FOR A NEW DOT PLOT" appears. The user must then reenter the desired dot plot specification.

\subsubsection{Euilding the Task}

DOTMGE, DOTOUT, and the required plotting subroutines are contained in MDLIB. which is distributed with Q. This library must be referenced in any user task build file if the task calls DOTMGE or DOTOUT.

The dot plot routines use a data base contained in the system common or system global. area (SGA) HCOMI; therefore, all. task build files for dot plotting tasks must contain one of the following COMMON specificatjons:

or

$$
\text { COMMON=HCOMI : RW: } 5 \text { (RSX-1ID) }
$$

$$
\mathrm{COMMON}=\mathrm{HCOM} 1: \mathrm{RW}: 6 \quad(\mathrm{RSX}-11 \mathrm{M})
$$

DOTOUT uses logical unit 3 (LUN 3) for plotting. It is not necessary to assign LUN 3 at task build time, but LUN 3 cannot be used for any other purpose by the task.

Exàmple:

; [122, 24] DOTTST.BLD 3-MAR-77 BUILD FILE FOR DOT PLOT TEST

OU : $[122,24]$ DOTTST, LS : $[122,24]$ DOTTST= IN : $[122,24]$ DOTTST.

IN : $[1,1]$ MDLIB/LB

1

$A S G=T I: 3$

LIBR=OTSCOR : RO COMMON=HCOMI : RW: 5 $1 /$.
; LUN3 USED FOR PLOTTING ;DO NOT USE FOR ANY OTHER PURPOSE ; LINK TO THE RESIDENT FORTRAN LIBRARY ; LINK TO SYSTEM COMMON (RSX-IID)

The pseudo-devices IN:, OU:, and LS: must be redirected to an appropriate disk DKn: before this task build file is run. 


\subsection{Keyboard Control of the system}

\subsubsection{HDO: Dot Plot Setup and Control}

HDO allows the user to set up and control a two-dimensional scatter plot on any Tektronix 4000 series terminal on the systern.

Forms :

\section{HDO> [term: ] [taskname] $=[$ uic]pltnam/sw HDO> [uic]pltnam/sw HDO>}

where term specifies a graphics terminal; taskname, the RAD50 name of the task that will produce the dot plot by calls to DOTOUT; uic, the dot plot owner; pitnam, a unique name assigned to the dot plot;. /sw, a switch or switches; and filename, the name of an indirect command file. Switches are optional. If the line contains an equals sign, any acceptable switch may be used on either side of the sign.

The following defaults are used for omitted parts of the line:

term the current terminal
taskname the task name used when the dot plot identified
by uic and pltnam was first set up. The task
name and plot name are required the first time a
particular dot plot is reguested.
The default terminal UIC, which is either the
logged-in UIC or the UIC specified with the /UI
switch in HPL.
pltnam The last dot plot specified at this terminal.

The following switches are available. The switches in the first set must be specified when setting up a new dot plot. Subsequently, they are optional.

$1: \ldots / E V: n$ specify the "event number", where $n$ is a decimal integer between $\emptyset$ and 255. This switch is used to control dot plot output. Only one dot plot, identified by the event number (see. IEVN in LOTOUT, Sec. 4.1.2), can be active at any one time.

2. /IN:I1:I2 specify data offsets, where "Il and I2 are decimal integers. Il and I2 are data indices for the horizontal and vertical axes, respectively. Il= 0 and $I 2=0$ point to the first word in the data buffer. Both Il and I2 must be specified if, this switch is present.

3. /UB:Ul:U2 Specify upper data value limits for the 
horizontal and vertical axes, respectively. $u 1$ and $\mathrm{u} 2$ can be positive or negative decimal in'tegers. Both $U l$ and $\mathrm{U} 2$ must be specified if this switch is present.

The switches in the second set are always optional.

1. /LB:Ll:L2 specify lower data value limits for the horizontal and vertical axes, respectively. Ll and L2 can be positive or negative decimal integers. Their default values are zero. Both L1 and L2 must be specified if this switch is present.

2. /MK:M1:M2 Specify the data masks for the horizontal and vertical axes, respectively. A logical . AND. is performed between $M I$ (or M2) and the data word pointed to by Il (or I2) to extract a field, and the number in this field is treated as an integer value whose low-order bit corresponus to the rightmost "one" bit in the mask. M1 and $M 2$ are octal numbers. Their default values are 177777 octal. Both Ml and M2 must be specified if this switch is present. A mask of zero is illegal.

Note: There is a bug in RSX-11D that will not accept input numbers larger than 15 bits. Therefore, the value 177777 octal should be input as -1 .

3. /NE:n specify the number of dots to output before quitting, where $n$ is a decimal integer. The default is an infinite number of points. A negative number indicates an infinite number of points.

4. ITI:n specify the number of seconds to plot before quitting, where $n$ is a decimal integer. The default is an infinite number of seconds. Zero indicates an infinite number of seconds.

5. $A B$ Abort the dot plot. This switch must be the only switch specified.

6. /RE Restart the previous dot plot. This switch must be the only switch specificd. It can be used to restart a plot that was stopped by the /AB switch or because of a timeout (/TI) or countout (/NE). If a plot that was stopped by a timeout or countout is restarted, the timeout and/or countout will also restart.

Once a switch value has been specified for a particular dot plot, it retains its value until explicitly changed. Switches may be continued onto another 1 ine by ending a command 1 ine wjth a comma $($,$) , as shown below.$

HDO>TT1: TEST= $[122,24]$ DOTPL1/EV $: 10 / \mathrm{IN}: 1: 20 / \mathrm{UB}: 200: 500$,

HDO $>7$ TI : 590 
1. The following command sets up a new dot plot on TT.2: HDO $>$ TT2 : DOTTST= $[122,24]$ PLOT $1 / \mathrm{EV}: 1 / \mathrm{IN}: 101: 194 / \mathrm{UB}: 1000: 50$, HDO>/LB: $50 \emptyset:-50 / \mathrm{MK}: 77: 177 / \mathrm{TI}: 5000$

Taskname (DOTTST) and switches EV, IN, and UB are mandatory. A grid is drawn on TT2 and points will appear when task DOTTST calls subroutine DOTOUT. After 50 bl seconds plotting will cease.

2. The following command changes the plot limits. HDO $>/ U B: 876: 53$

A new grid will be drawn on TT2.

3. The following command changes the data being plotted. HDO>PLOT1/IN: $: 1 \%$ UB: $100: 200 / \mathrm{LB}: \emptyset: \emptyset / \mathrm{MK}: 177: 377$

A new grid will be drawn on TT2.

4. The following command aborts the plot. $\mathrm{HIDO}>/ \mathrm{AB}$

5. The following command restarts the plot. HDO $>$ PLOT1/RE

In this case, a new grid will not be drawn. 


\section{ACKNOWLEDGMENTS}

We are indebted to Richard kitteli and Randy Jeppeson, who designed and implemented the first versions of the histograra plot and dot plot systems, and to Mireille Pahud, who wrote the original package of histogram plotting subroutines. We would also especially like to thank janis Builta, Elvira Martinez, ana Lorrie voorhees for all their efforts in the preparation of this manual. 
INDEX

Bin size . . . . . . . . 8

Clear .......... . 13

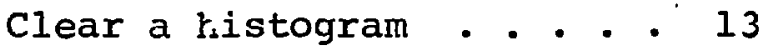

Clear all histograms . . . 13

Create a histograin . . . . . 9

Data entry . . . . . . . 10

Data index . . . . . . . 7

Delete . . . . . . . 14,15

Delete a histogram . . . 14,15

Delete all histograms . . 15

Descriptor array . . . . 5,10

Dimension ........ . 7

Disk histograms . . . . . . 2

Dot plot management . . . 22

Dot plot output . . . . . 22

Dot plot setup and control - 25

Dot plotting . . . . . . 22

DOTMGE . . . . . . . . 22,23

DOIOIT . . . . . . . . 22,23

Enter dzta into a histogram 10

Flush histogrims . . . . 12

HBE •. . . . . . . . . 19

HCOMI . . . . . $17,23,24$

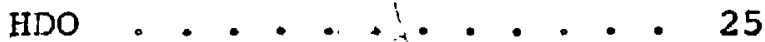

HIS . . : . . . . . 18

HISTCA - + * . . . . 13

HISTCI. • ० • . . • • • 13

HTSTDE.......... . 15,16

HISTIE .......... . 14,16

HLETEN e . . . . . 10,16

HISTFL •. . . . . . . 12,16

Histogram manager . . . . 17

HISTRE . . . . . . . . 10

HISTSU . . . . . . . . 9,15

HOF . . . . . . . 17,18

HPI • . . . . . . . . . 19

HSTMGE •. . • • • • . . 17
IDHA • . . . . . . . 5

IEVBUF ............ 23

IEVN • • . . . . . . . . 23

Initialize display system . 19

List histogram descriptor

array ........ . 18

Logical unit $3 . . . . .24$

Iost memory . . . . . . . 17

Lower limit . . . . . . . 8

LUN 3 . . . . . . . . 24

Mask •. . . . . . . 7

MDLIB library . . . . 17,24

Memory allocation .. . 2,16

Name • . . . : . . . . 6

Negative data values . . . 8

Number . • • • • • • . 6

Number of bins . . . . . 8

Number of parameters . . . 7

Overflows ....... 2,13

Owner . . . . . . . . 6

Plot a histogram . . . . 19

Receive AST . . . . . . 23

Retrieval . . . . . . 10

Retrieve data from a

histogram . . . . . 10

Scatter plot . . . . . . 22

Set up a histogram . . . . 9

Set up dot plot . . . . . 25

Stop histogramming . . . 18

System common . . . . . 17

System global area . . . 17

Turn off histogramming . - 18 